\title{
Saúde sexual e reprodutiva e Enfermagem: um pouco de história na Bahia
}

\author{
Sexual and reproductive health and Nursing: a bit of history in Bahia \\ Salud sexual y reproductiva y Enfermería: un poco de historia en Bahía
}

\author{
Joise Magarão Queiroz Silva', Patrícia Figueiredo Marques", Mirian Santos Paiva"II \\ ' Universidade Federal da Bahia, Escola de Enfermagem (Mestranda). Salvador-BA, Brasil. \\ "Universidade Federal do Recôncavo da Bahia, Centro de Ciências da Saúde, \\ Curso de Graduação em Enfermagem. Santo Antônio de Jesus- BA, Brasil. \\ II' Universidade Federal da Bahia, Escola de Enfermagem, Departamento de Enfermagem Comunitária. Salvador-BA, Brasil.
}

\author{
Submissão: 20-05-2011 Aprovação: 16-06-2013
}

\section{RESUMO}

O estudo objetivou analisar a inclusão dos conceitos de saúde sexual e reprodutiva nos currículos da Escola de Enfermagem da Universidade Federal da Bahia, de 1972 a 2006. Utilizou a abordagem qualitativa, a pesquisa documental e a entrevista semiestruturada com docentes em regime de dedicação exclusiva. Os resultados revelaram que, inicialmente, as disciplinas eram exclusivamente voltadas aos aspectos biológicos da saúde da mulher, enfocando apenas a condição materna. Os conceitos de saúde sexual e reprodutiva, na perspectiva feminista, foram introduzidos posteriormente, para atender demandas de políticas de formação de mão-de-obra na área de saúde, representando também uma ampliação na compreensão da condição social da mulher. Conclui-se que ocorreu uma evolução nos currículos com a introdução desses conceitos, principalmente a partir da década de 1990.

Descritores: Enfermagem; Ensino; Saúde Sexual e Reprodutiva.

\section{ABSTRACT}

The study aimed to analyze the introduction of sexual and reproductive health concepts in the curriculums of nursing course of the Federal University of Bahia, from 1972 to 2006. It was carried out through qualitative approach, documentary research and semi-structured interview with professors who work exclusively for the institution. The results showed that, initially, the academic disciplines were exclusively related to biological aspects of women's health, focusing only on the maternal condition. The concepts of sexual and reproductive health in the female perspective were introduced later, in response to political demands for the creation of labor force in the field of healthcare with commitment to women's social condition. It was concluded that the curriculums evolved with the introduction of such concepts, especially from the decade of 1990's on.

Key words: Nursing; Teaching; Sexual and Reproductive Health.

\section{RESUMEN}

El estudio ha objetivado analizar la inclusión de los conceptos de salud sexual y reproductiva en los currículos de la Escuela de Enfermería de la Universidad Federal de Bahía, desde 1972 hasta 2006. Ha utilizado un abordaje cualitativo, la pesquisa documental y la entrevista semi estructurada con docentes de dedicación exclusiva. Los resultados han revelado que, inicialmente, las asignaturas eran exclusivamente vueltas a los aspectos biológicos de la salud de la mujer, enfocando apenas la condición materna. Los conceptos de salud sexual y reproductiva, en la perspectiva feminista, fueran introducidos posteriormente, para atender las demandas de políticas de formación de mano de obra en el área de salud que prestase una atención sensibilizada a la condición social de la mujer. Se concluye que ha ocurrido una evolución en los currículos con la introducción de eses conceptos, principalmente a partir de la década de 1990.

Palabras clave: Enfermería; Enseñanza; Salud Sexual y Reproductiva.

\section{AUTOR CORRESPONDENTE Patrícia Figueiredo Marques E-mail: pfmenf@yahoo.com}




\section{INTRODUÇÃO}

No Brasil, as discussões sobre a sexualidade e a reprodução foram institucionalizadas a partir da década de 1950 do século XX, período em que se manifestaram preocupações governamentais significativas com o crescimento populacional, dada sua possível interferência no crescimento econômico. Desse momento em diante, nas décadas de 60 e 70 do mesmo século, houve maior investimento do governo brasileiro com relação à formulação de programas para o controle da natalidade, sob a forma de planejamento familiar. Tais programas não se preocupavam com os direitos sexuais e reprodutivos das mulheres e dos homens $\mathrm{s}^{(1-3)}$.

A partir da década de 80 , com a intensificação das lutas do movimento de mulheres, a Reforma Sanitária e a redemocratização do país, as questões relacionadas aos diretos sexuais e reprodutivos passaram a integrar a pauta das discussões nas conferências nacionais de saúde. A mudança de atitude governamental quanto à perspectiva social e ampliada da saúde ocorreu em um momento de abertura política, favorável à conquista de direitos. Nesse período, a saúde passou a compor o quadro dos problemas sociais para os quais os movimentos organizados da sociedade civil reivindicavam soluções ${ }^{(1-3)}$.

O reconhecimento da fragmentação da assistência à saúde da mulher, denunciada pelas feministas, passou a ser compartilhado por sanitaristas, demógrafos, cientistas sociais e militantes de partidos políticos. Mediante a pressão do movimento de mulheres e baseado em algumas das reivindicações na atenção a saúde, em 1984 o Ministério da Saúde lançou o Programa de Assistência Integral à Saúde da mulher (PAISM) ${ }^{(4)}$.

Neste Programa, a Enfermagem ampliou seu espaço de atuação, com a possibilidade de desenvolver atividades educativas, consultas de enfermagem e demais ações próprias da atenção primária, nos serviços de pré-natal e planejamento familiar. Na rede hospitalar, sua atuação voltou-se para o alojamento conjunto, a administração dos centros obstétricos e a realização do parto, quando habilitadas.

Na década de 1990, houve um redirecionamento das políticas internacionais para questões do meio ambiente e do desenvolvimento, culminando na Conferência Internacional de População e Desenvolvimento (CIPD), realizada no Cairo em $1994^{(5)}$. A CIPD conferiu papel primordial à saúde e aos direitos sexuais e reprodutivos, superando o enfoque puramente demográfico. O novo foco provocou uma transformação profunda no debate sobre o crescimento populacional ao dar prioridade às questões dos direitos humanos.

A CIPD foi um marco importantíssimo no que se refere a saúde sexual e reprodutiva, pois trouxe à tona esses conceitos de forma mais abrangente ao discuti-los numa perspectiva que ultrapassava os limites do biológico e a obrigatoriedade da reprodução para as mulheres. Com isso, possibilitou mudanças no âmbito governamental e político vigente, em que a maioria dos países desenvolvia programas verticais com ênfase no ciclo gravídico-puerperal(5).

Muitas das sugestões e dos compromissos assumidos na Conferência do Cairo repercutiram e respaldaram as discussões na IV Conferência Mundial sobre a Mulher, realizada em
Beijing em 1995. Houve avanços na definição dos direitos sexuais e reprodutivos, que passaram a ser vistos como direitos humanos ${ }^{(6)}$. Os direitos sexuais foram definidos de maneira mais autônoma em relação aos direitos reprodutivos, dissociando a sexualidade da reprodução. A igualdade de gênero foi outro tema bastante relevante no que tange às discussões sobre saúde sexual e reprodutiva.

Em ambas as Conferências, os governos de vários países, entre os quais o Brasil, assumiram o compromisso de levar em conta os direitos sexuais e reprodutivos ao elaborar e implementar as políticas e os programas nacionais dedicados à população e ao desenvolvimento, em especial os de planejamento familiar. Os princípios de Cairo e Beijing opõem-se radicalmente à imposição de metas populacionais conceptivas ou contraceptivas que não sejam pautadas no livre arbítrio e na autonomia sobre o corpo, reconhecendo para isso o fim de todas as formas de discriminação(6).

Nesse contexto de mudanças conceituais, em que surge uma nova perspectiva sobre a saúde da mulher, é de grande relevância pesquisar como se deu a inclusão dos conceitos de saúde sexual e reprodutiva nos currículos do curso de enfermagem da Universidade Federa da Bahia, um fenômeno que repercutiu no perfil dos enfermeiros por ela formados. Como conceitos transversais a todas as áreas de atenção a saúde, considera-se que sua inclusão na formação em Enfermagem contribui para o exercício dos direitos humanos ${ }^{(7)}$.

Neste sentido, esse estudo propôs-se a responder à seguinte questão: Como se deu a inclusão dos conceitos de saúde sexual e reprodutiva nos currículos da Escola de Enfermagem da Universidade Federal da Bahia (EEUFBA)? Seus objetivos foram analisar a inclusão dos conceitos de saúde sexual e reprodutiva nos currículos da Escola; identificar as disciplinas do currículo em que são adotados e descrever como os usos desses conceitos foram e são abordados na teoria e na prática.

\section{PROCESSO METODOLÓGICO}

Para a apreensão do objeto e o alcance dos objetivos propostos, utilizou-se a abordagem qualitativa, que oportunizou a análise, a descrição e a compreensão do problema a partir de suas características específicas. O propósito foi obter informações significativas para auxiliar no processo de desvendamento da temática de forma concisa, clara e crítica.

A abordagem qualitativa apresentou-se como a mais apropriada para empreender a construção de conhecimento sobre a historicidade, as contradições e as possíveis conexões existentes entre os elementos que constituem o fenômeno em análise. Para melhor apreender o objeto de estudo, a investigação foi desenvolvida em duas etapas interdependentes e concomitantes: a pesquisa documental e a escuta de protagonistas do processo.

Entende-se por pesquisa documental o estudo desenvolvido sobre material elaborado previamente e que ainda não tenha recebido tratamento analítico. No presente estudo, a fonte do material empírico foi composta por documentos de primeira mão, que não haviam passado por análise prévia ${ }^{(8)}, \mathrm{o}$ que incluiu os currículos de enfermagem da EEUFBA de 1972 
a 2006. A escolha desses períodos ocorreu pela influência dos Programas de Atenção à Saúde da Mulher, inicialmente na década de 1970, quando ocorreu a implantação do Programa Materno-infantil, e em 2004, quando houve a reorganização das políticas de saúde mulher, no PNAISM.

Também foram realizadas e gravadas entrevistas semiestruturadas com docentes de dedicação exclusiva que lecionavam as disciplinas que abordavam os conceitos de saúde sexual e reprodutiva. No período da realização da pesquisa, o curso contava com dez docentes de dedicação exclusiva, sendo uma delas a orientadora do estudo. Uma negou-se a participar e outra disse não ter tempo disponível, de tal modo que as entrevistas foram realizadas com sete docentes. A abordagem foi viabilizada com a entrega de uma carta-convite e do resumo do projeto a cada uma das docentes, a fim de agendar a data para realização das entrevistas, que foram realizadas nas salas das docentes. Posteriormente, as entrevistas foram transcritas e o material resultante foi arquivado em mídia para ser preservado por cinco anos.

Não foi possível analisar os programas das disciplinas de todos os anos estabelecidos, pois alguns documentos não foram encontrados. Entretanto, isto não comprometeu o estudo, já que grande parte foi localizada e organizada por décadas. Os dados foram analisados mediante a Análise Temática proposta por Minayo ${ }^{(9)}$.

Os aspectos éticos foram compreendidos não apenas como uma questão de sigilo, abrangendo também o anonimato, o consentimento informado e a liberdade dos participantes retirarem-se do processo sem qualquer prejuízo, conforme preconiza a Resolução 196/96 sobre pesquisa com seres humanos. O projeto de pesquisa foi registrado no Sistema Nacional de Ética na Pesquisa e ao Comitê de Ética em Pesquisa da Instituição, tendo sido aprovado mediante parecer de $n^{\circ}$ 062/2007. Para manter o anonimato, as entrevistadas receberam pseudônimos de flores: Girassol, Copo de Leite, Gardênia, Margarida, Rosa Amarela, Tulipa e Angélica. Todas assinaram o termo de consentimento livre e esclarecido.

\section{RESULTADOS E DISCUSSÃO}

No período estudado houve três currículos: 1972-1993 (parecer 163/1972); 1994-1995 (Parecer 314/1994) e 19962006 (Parecer 472/1995). A evolução dos conceitos na saúde da mulher tem início com a saúde materna e isto pode ser observado na estrutura do currículo da graduação em enfermagem sob a vigência do parecer 163/1972.

Consta no Quadro 1 as disciplinas que abordavam a saúde da mulher e cujos conteúdos diziam respeito unicamente ao ciclo gravídico-puerperal. A saúde reprodutiva e o sexo eram entendidos apenas no sentido biológico, assim como os problemas correlatos: frigidez, fertilidade e infertilidade. Em seu estudo, Lessa $^{(10)}$ aponta que as disciplinas sobre a assistência à saúde da mulher desse período preocupavam-se apenas com o aparelho reprodutor feminino, "o aparelho biológico-sexual da mulher".

Esse currículo vigorou até 1993. No início da década de 1980, deixaram de existir as habilitações em obstetrícia e em saúde pública e o curso foi renomeado Bacharelado em Enfermagem.

Quadro 1 - Características das disciplinas sobre saúde sexual e/ou saúde reprodutiva nos currículos da graduação em enfermagem da EEUFBA,1972 a 2006. Salvador, Bahia, 2008.

\begin{tabular}{|c|c|c|c|}
\hline Década & Disciplinas & Carga horária & Pré-requisito \\
\hline 1970 & $\begin{array}{l}\text { Enf.-144 Enfermagem materno-infantil } \\
\text { Obstetrícia I }\end{array}$ & $\begin{array}{ll}\text { T-90 } & \text { P-270 } \\
\text { T-45 } & \text { P-90 }\end{array}$ & Enf.-135 Enfermagem médico- cirúrgica \\
\hline 1980 & $\begin{array}{l}\text { Enf.- } 144 \text { Enfermagem materno-infantil: } \\
\text { Enfermagem Obstétrica Enfermagem Pediátrica }\end{array}$ & $\begin{array}{l}360 \text { total } \\
\text { T-45 P- } 90 \text { E-45 cada uma. }\end{array}$ & Enf.-135 Enfermagem médico-cirúrgica \\
\hline 1990 & $\begin{array}{l}\text { Enf.- } 144 \text { Enfermagem materno-infantil } \\
1995 \text { - Enf. 157-Enfermagem na assistência } \\
\text { integral à saúde da mulher } \\
\text { 1996/1999 Enf.-009 Enfermagem na } \\
\text { atenção à saúde da mulher I } \\
\text { Enf.-020 } \\
\text { Enfermagem na atenção à saúde da mulher II }\end{array}$ & $\begin{array}{l}360 \\
180 \text { T-90 P-- } 90 \\
120 \text { T-60 P-60 } \\
60 \text { T-30 e P- } 30\end{array}$ & $\begin{array}{l}\text { Enf.-135 Enfermagem médico-cirúrgica } \\
\text { Enf. } 008 \text { Enfermagem clínico-cirúrgica }\end{array}$ \\
\hline 2000 & Enf. 161 Enfermagem na atenção à saúde da mulher & $\begin{array}{l}180 \text { T- } 90 \text { P-90 (até 2003) } \\
204 \text { T- } 100 \text { P-104 (A partir de 2004) }\end{array}$ & Enf. 008 Enfermagem clínico-cirúrgica \\
\hline
\end{tabular}

$T=$ Teórica $P=$ Prática

FONTE: Documentos das estruturas curriculares da EEUFBA. 
A partir da década de 1980, os conceitos de saúde sexual e reprodutiva passaram a ser abordados no contexto político, econômico, cultural e social que determina a situação da muIher na sociedade. Esse fato pode ser verificado pela presença dos conteúdos: política de assistência integral à saúde da muIher, papel da mulher na sociedade, fertilidade e infertilidade, assistência à mulher no planejamento familiar.

A preocupação com a modernização do currículo de Enfermagem ocorreu por influência do movimento feminista no final da década de $1980^{(10)}$. O feminismo, como ideologia política, pode ser identificado desde o século XIX, mas foi nas décadas de 70 e 80 do século XX que o pensamento feminista ingressou no campo acadêmico e impõe-se como uma tendência teórica inovadora e de forte potencial crítico $^{(11)}$.

Em 1983, o processo de implementação do PAISM pelo Ministério da Saúde ampliou essas discussões. Deve-se registrar que na EEUFBA a disciplina Materno-infantil já trazia conteúdos sobre a saúde da mulher. Em 1988, as propostas feministas foram reafirmadas e consolidadas no âmbito da EEUFBA com a criação do GEM $^{(11)}$.

Uma das docentes também destacou o papel do GEM no processo de inclusão nas disciplinas dos conceitos de saúde sexual e reprodutiva:

[...] Nós começamos o processo da disciplina com uma perspectiva feminista desde 1987, quando o GEM foi criado, o grupo foi criado com a perspectiva feminista e nós tínhamos a preocupação de que as alunas da graduação tivessem práticas diferenciadas [...] (Copo de Leite)

Na década de 1990, os conceitos de saúde sexual e reprodutiva foram ampliados com a introdução da categoria gênero, instrumento útil para compreensão da situação da mulher na sociedade. A grande maioria das entrevistadas concordou que esses conceitos vêm sendo abordados na EEUFBA desde o início da década de 1990; enquanto uma pequena parte acredita que tenham sido abordados desde a sua proposição nos fóruns nacionais e internacionais, aprimorando-se ao longo dos anos, principalmente a partir do final da década de 1980 e início da década de 1990.

[...] Esse conteúdo começou a ser introduzido na década de 90 aqui na nossa disciplina; inclusive nossa Escola é pioneira não só nesse conteúdo de saúde sexual e reprodutiva, mas também na forma como ele vem sendo dado. Nós damos os conteúdos de forma didática, pois o aluno vai estudar procurando ver sua fisiologia, sua anatomia, o funcionamento, a prevenção, a promoção da saúde. Também temos trabalhado com oficinas onde o conceito de gênero permeia esses conteúdos[...] (Margarida)

No final dos anos 1990, a disciplina Enfermagem na atenção à saúde da mulher estabeleceu como conteúdo a assistência à saúde sexual e reprodutiva. Antes essa temática era vinculada a discussões advindas dos programas públicos da área técnica de saúde da mulher do Ministério da Saúde: PAISM, DST/AIDS, planejamento familiar, prevenção e detecção ao câncer de colo uterino e mama, pré-natal. A maioria das docentes entrevistadas considera que teoria e prática estão interligadas, sendo muitas vezes influenciadas pela dinâmica dos serviços sem, contudo, se limitar a ela.

Ainda conforme Parecer 314/1994, no currículo vigente no período de 1994-1995, as docentes buscavam implementar no processo de ensino e aprendizagem a percepção da mulher como cidadã, inserida numa sociedade caracterizada pela reprodução de desigualdades de gênero. Buscavam ainda desenvolver e ensinar uma prática diferenciada visando ao empoderamento das mulheres, oferecendo aos estudantes as condições necessárias para a conscientização e fazendo com que as mulheres criassem meios de decidir sobre si mesmas.

...as estudantes aprendem sempre que há oportunidades de colocar a mulher como sujeito da atenção e instrumentalizá-la para tomar decisões sobre sua vida... (Girassol)

...nós procuramos ensinar aos alunos para que eles forneçam as informações necessárias para que as mulheres tomem as decisões... (Copo de Leite)

Para as docentes entrevistadas, as práticas desenvolvidas no ciclo gravídico-puerperal envolvem o pré-natal, o alojamento conjunto, a atuação em centro obstétrico, a prevenção de câncer cérvico-uterino e de mama e o planejamento familiar. Algumas também citaram o cuidado de enfermagem no climatério e na menopausa, além da assistência à mulher idosa. Duas mencionaram a atividade educativa e as oficinas para o incremento das práticas assistenciais. Ao fazer referência às práticas, as docentes destacaram a falta de insumos, especialmente de métodos contraceptivos, como elemento que prejudica a continuidade da atenção.

No currículo de 1971-1993, as disciplinas relacionadas à saúde reprodutiva deixaram de ser fragmentadas como eram desde a fundação da EEUFBA, em 1947. Exemplo disso está na disciplina Saúde materno-infantil, que era dividida nas disciplinas Obstetrícia I e II. A primeira era ministrada durante a graduação e somente os alunos que optavam pela habilitação em obstetrícia cursavam a Obstetrícia II. Os que optavam por outras habilitações não tinham acesso aos conteúdos da segunda disciplina, o que fragmentava o conjunto de conteúdos referentes à assistência à saúde da mulher, contribuindo para a fragmentação da integralidade da pessoa assistida. Segundo Christófaro $^{(12)}$ :

...a flagrante compartimentalização e a minimização do conhecimento estabelecidas na legislação em vigor resultam em um processo de formação anacrônico onde o enfermeiro "aparece" em falsas e frágeis vertentes: habilitação geral e habilitações especificas. Sobre este aspecto, o que as atuais normas produzem, de fato, é a indefinição do profissional. As chamadas habilitações específicas (por área), cujo oferecimento é opcional para a Instituição e para os alunos, tornaram a formação do enfermeiro um processo pulverizado em detrimento de um processo que privilegie as bases técnico-científicas da profissão. 
No material documental analisado não foram identificadas ações para implementação da interdisciplinaridade nos currículos vigentes entre 1972 e 2006 da EUEFBA, em que pese a importância crescente das discussões sobre as inter-relações entre diversos conteúdos para compreensão do mundo, presentes nos currículos da Instituição desde a década de 1960.

A partir da década de 1980 a prática era dividida em blocos separados por campos e docentes com um determinado número de alunos (seis discentes por uma docente). Todas as estudantes passavam por todos os campos de forma contínua, sem repetir a prática ou o programa de saúde. As docentes procuravam fazer com que tivessem uma visão ampliada das questões construídas socialmente e culturalmente, abordando todos os conteúdos com enfoque de gênero, numa perspectiva feminista.

No processo de transição da década de 1990 e o início dos anos de 2000, observa-se que as ementas passam a incluir a temática de gênero, inicialmente articulada com a classe social e, em seguida, com a raça/etnia. Isto marca as discussões sobre o processo saúde-doença das mulheres quando não relacionado à reprodução. Além disso, as ementas aparecem mais descritivas, detalham um número maior de agravos à saúde da mulher e abordam a sexualidade a partir de transtornos e desvios. A temática do aborto só apareceu nas ementas na década de 1990, mesmo sendo um grave problema de saúde pública no município de Salvador.

O processo de ensino e aprendizagem, que já era influenciado pelas propostas de Paulo Freire ${ }^{(13-14)}$, apropriou-se da pedagogia feminista ${ }^{(15)}$. Observa-se mais uma vez a influência do GEM e do feminismo para transformação dessas práticas, o que contribuiu de forma significativa para construção de uma metodologia inovadora, baseada nos conceitos de gênero e raça/etnia e voltada para uma atenção humanizada. A disciplina de Atenção a saúde da mulher, vista até então de forma biológica, passa a adotar uma visão da mulher como um todo, nos aspectos reprodutivos, sexuais, psicológicos e sociais.

As práticas feministas têm similaridades com a metodologia criada por Paulo Freire, em que o sujeito e suas experiências são colocados como centro, sendo assim uma pedagogia transformadora e humanizadora. O movimento feminista, desde seus primórdios na década de 60 até os dias atuais, têm tido uma visão diferenciada no que diz respeito às questões de gênero, defendendo os direitos das mulheres e principalmente lutando por sua emancipação cultural, política e social. Enfatiza a relação constante entre as identidades pessoal, social e coletiva, tendo como base que a identidade pessoal é informada pelos padrões de gênero, o que influi na identidade social e coletiva das mulheres ${ }^{(15)}$.

O próprio movimento feminista vem sofrendo várias influências, desde o marxismo, o estruturalismo, o pós-estruturalismo ou o funcionalismo e também de diferentes disciplinas da área das ciências humanas, como sociologia, antropologia, psicanálise, pedagogia, psicologia e filosofia.

A construção de aprendizado é feita por meio de práticas educativas diferenciadas, em que o corpo é um dos temas centrais. Ao tratar desde tema, as docentes reconhecem o desconhecimento que as mulheres têm sobre seus próprios corpos e suas implicações ${ }^{(16-17)}$. Uma docente enfatiza a importância dessas práticas não só na formação das graduandas de enfermagem, como também dos demais profissionais da saúde:

[...] nós tínhamos a preocupação de que as alunas da graduação tivessem práticas diferenciadas, no primeiro momento fazíamos o treinamento das estudantes com práticas educativas, essas práticas eram práticas feministas já desenvolvidas por outras ONGs e também nós fazíamos práticas no Serviço de Saúde de acompanhamento. Essa prática não diz respeito apenas à formação dos estudantes, mas também a formação dos profissionais de saúde [...] (Copo de leite)

O movimento feminista criou uma nova metodologia para a ação educativa, fortemente ancorada na perspectiva de gênero, com ênfase nas atividades em grupo e metodologia que articula a racionalidade e a subjetividade, além de experiências, conhecimentos técnicos e teóricos. Dentre as atividades educativas, são utilizadas principalmente as oficinas, que propiciam a construção do conhecimento de forma diferenciada, além de outros processos mais horizontais e democráticos, em todos os envolvidos participam de forma direta de tudo que é realizado ou construído ${ }^{(15)}$.

A partir da análise documental realizada observou-se que em algumas disciplinas principalmente entre as décadas de 1970 e 1980 ainda há um olhar predominante para os aspectos biológicos da mulher e a atenção ao ciclo gravídico-puerperal. Tal como afirmam Tyrell, Santos, Lucas ${ }^{(18)}$, a assistência à saúde da mulher é limitada ao ciclo reprodutivo e, mais especificamente, à área obstétrica, permanecendo a ênfase no período reprodutivo e na "naturalização" das relações de poder entre os sexos.

A partir do final da década de 1980 começa a surgir um olhar diferenciado, buscando superar a atenção voltada unicamente ao ciclo gravídico-puerperal, que se intensifica nas décadas de 1990 e 2000. As metodologias de ensino também apresentam forte evolução e as aulas puramente expositivas são substituídas por seminários, simpósios, visitas domiciliárias e atividades educativas, entre elas as oficinas.

Na década de 1970, a disciplina Enfermagem e Obstetrícia I tinha uma carga horária total de 135 horas, distribuída em 45 horas teóricas e 90 horas práticas, distribuição essa que privilegia a prática em detrimento da teoria, reforçando uma ideologia de "naturalidade" para o cuidar nessa profissão feminina. Isso persiste na década de 80, com 135 horas distribuídas entre a prática e o estágio e apenas 45 horas para a teoria. Na década de 90, especialmente a partir de 1995, começa a haver maior preocupação com a teoria, com a inclusão da disciplina Enfermagem na Atenção Integral à Saúde da Mulher, em que se igualam as cargas horárias da teoria e da prática (90 horas), o que permaneceu até 2003.

Já na disciplina Enfermagem na Atenção à Saúde da MuIher, a partir de 2004, volta a privilegiar a prática em detrimento da teoria, mas de forma bem menos significativa (Quadro 1). Dispensar maior tempo para a prática pode demonstrar uma preocupação em formar profissionais cada vez mais técnicos, com uma visão limitada e fragmentada da assistência. 
Isso só começa a mudar a partir da década de 1990, quando se passou a formar profissionais mais capacitados para prestar uma assistência integral e mais humanizada.

A partir da década de 1990, os conceitos de saúde sexual e reprodutiva começam a se expandir com a introdução de conteúdos ancorados na perspectiva de gênero, conceitos mais aprofundados de saúde sexual e reprodutiva, com a finalidade de empoderamento das mulheres para tomar decisões e lutar por seus direitos, como pode ser observado na fala de algumas docentes:

[...] essa disciplina procura adequar à forma de atendimento, de que a mulher tenha a expressão de sua própria voz, que seja autônoma com relação as suas decisões[...] (Copo de Leite )

[...] Dentro desses temas sempre são abordados na perspectiva da saúde e na qualidade da saúde da mulher, o posicionamento da mulher mais ativa, da mulher mais empoderada, da mulher mais consciente do seu papel[...] (Gardênia)

A disciplina relacionada à saúde da mulher sofreu várias modificações de nomenclatura até atingir a atual denominação, ou seja, Enfermagem na Atenção à Saúde da Mulher. As mudanças não são apenas de nomenclatura, mas se fazem presentes nas ementas, nas metodologias e nos conteúdos. Exemplo disso é que, na década de 1970, estes estão voltados para a obstetrícia, envolvendo o ciclo gravídico-puerperal e algumas questões ginecológicas relacionadas a possíveis intercorrências no processo reprodutivo. Na década de 1980 são incluídas discussões sobre a política de assistência integral à saúde da mulher, o papel da mulher na sociedade, a fisiopatologia nas diversas fases do ciclo vital da mulher, fertilidade, infertilidade e assistência no planejamento familiar. Na década de 1990 e no início dos anos 2000, ocorre a inclusão da temática gênero e discussões sobre aborto, DST/AIDS, transmissão vertical do HIV, os programas de saúde da mulher nos níveis nacional e internacional, os perfis demográfico e epidemiológico, indicadores de avaliação e acompanhamento da atenção a saúde da mulher, câncer cérvico-uterino e de mama e doenças associadas ao ciclo gravídico-puerperal por causas diretas e indiretas.

Até a década de 1980, as disciplinas vigentes eram voltadas ao ciclo gravídico-puerperal. A partir da segunda metade da década de 1980, por influência das políticas de saúde, a disciplina mudou seu enfoque para a assistência à mulher e à criança, embora seus conteúdos tenham permanecido voltados para fase reprodutiva. No final dessa década, por influência do movimento feminista na política de saúde, seu conteúdo passou a focalizar a mulher de forma mais abrangente.

Atualmente as questões de gênero são bem mais trabalhadas dentro da disciplina, que é norteada pelo enfoque de gênero e a perspectiva feminista.

[...]tanto em consulta como hospitalar e assistência ao parto às mulheres no parto e atividades educativas na perspectiva de gênero e feminista[...] (Angélica)
[...] toda a disciplina em si aborda as questões de gênero e aborda questões relacionadas a saúde sexual e alguns enfoques da disciplina prezam pela questão da saúde reprodutiva, mas os dois conteúdos eles estão interligados e eles são entendidos de um modo geral em todo contexto da disciplina...(Rosa Amarela)

A visão da maioria das docentes entrevistadas é que os conceitos são trabalhados em toda a disciplina, tanto na teoria como na prática. Os documentos mostraram que realmente houve uma evolução desses conteúdos com maior ampliação a partir do final da década de 80 e início da década de 90.

\section{ALGUMAS REFLEXÕES FINAIS}

A EEUFBA até 2006 ainda estava sob a vigência do currículo aprovado pelo parecer 472/95 que é norteado pelo parecer 314/94, sendo que Diretrizes Curriculares para os cursos de Enfermagem foram aprovadas após o parecer 1133 do CNE/ CES em 7/08/2001. Muito do que foi estabelecido nas Diretrizes já foi incluído no currículo que estava vigente, como o incentivo à pesquisa e à extensão, além do trabalho de conclusão de curso de graduação.

Desde sua criação, o GEM vem exercendo forte influência nas teorias e práticas, tendo sido por sua vez influenciado pelo movimento feminista. Os conteúdos sobre saúde sexual e reprodutiva são observados nas disciplinas: Obstetrícia I e II, Ginecologia, Enfermagem nas Doenças Sexualmente Transmissíveis, Enfermagem Materno-infantil, Enfermagem na Atenção Integral à Saúde da Mulher, Enfermagem na Atenção a Saúde da Mulher I e II e Enfermagem na Atenção a Saúde da Mulher. Desde a década de 1980 até a atualidade, tais disciplinas receberam várias influências: o feminismo, o GEM, o PAISM e a reforma sanitária, além de reflexões internas à profissão.

Os conteúdos disciplinares são abordados por meio de oficinas, atividades educativas, conteúdos teóricos. Na prática, estão relacionados à assistência às mulheres no pré-natal, no planejamento familiar, no alojamento conjunto, no centro obstétrico e na prevenção de câncer de colo de útero e mama. Ambos são norteados pelo enfoque de gênero e a perspectiva feminista para que as mulheres sejam empoderadas e tomem decisões sobre seus corpos.

A evolução desses conceitos foi perceptível a partir da década de 1970, ocasião em que eram direcionados para aspectos biológicos do ciclo gravídico-puerperal, e a partir da década de 1980, quando começaram a ocorrer mudanças. Isto é mais evidente após a criação do PAISM (1984), que ampliou a atenção para além da noção reducionista da reprodução como único aspecto a ser observado na saúde da mulher. $\mathrm{O}$ programa também incorporou nas práticas em saúde o ideário feminista favorável à emancipação das mulheres. Um de seus pontos principais era o planejamento familiar, fundado no princípio de que toda mulher tem livre escolha e o direito de decidir sobre ter ou não filhos.

Os currículos e demais documentos das disciplinas analisados guardam relação com as falas das docentes entrevistadas e com o direcionamento dado à proposta de ensino do 
curso de graduação em Enfermagem da UFBA, em resposta às demandas de formação na área de saúde. Num primeiro momento, até início dos anos 1980, sem influências das reflexões oriundas do movimento de mulheres e depois absorvendo suas ideias e propostas, especialmente após a constituição do GEM, grupo de pesquisa com esse enfoque.

Outra reflexão é a ausência da transversalidade dos conceitos de saúde sexual e reprodutiva no currículo, o que reproduz uma visão fragmentada de cuidado, em que uma disciplina pode ser o único espaço de discussão sobre a temática e as demais não resgatam nem comentam o assunto. Isso compromete a possibilidade de ações integrais na saúde da mulher e dificulta o exercício da interdisciplinaridade. Consequentemente, o currículo contribui para a formação de profissionais que reproduzem em sua prática a falta de integralidade na atenção a mulheres e homens, quaisquer que sejam sua classe, raça, etnia, geração, restringindo-se a aspectos biológicos.

Em síntese, ao analisar os documentos, foi observado que os conceitos de saúde sexual e reprodutiva só aparecem nas disciplinas que dizem respeito a saúde da mulher e, em especial, a partir da década de 1980, com exceção na disciplina Enfermagem nas doenças transmissíveis, que abordava as "doenças venéreas", desde o final da década de 1970. Entretanto, essa disciplina deixou de fazer parte do currículo após o parecer 314/1994, que aprovou o currículo de 1995. Nas demais disciplinas, a evolução desses conceitos ocorreu de forma gradativa e novos conteúdos foram sendo incorporados. Tais conteúdos são essenciais para formação do profissional de saúde, uma vez que possibilitam lidar com diversas questões sociais, culturais e de gênero, garantia para uma prática séria e humanizada.

Foram observadas lacunas de conteúdo que fazem parte da temática da saúde sexual e reprodutiva, como a discussão sobre a sexualidade e a reprodução de mulheres homossexuais, bissexuais e transexuais, mulheres indígenas e trabalhadoras da zona rural e as repercussões do HTLV no ciclo gravídico-puerperal. Também há pouco aprofundamento sobre como as profissionais de enfermagem devem lidar com questões relativas à sexualidade.

Pode-se dizer que os conteúdos detalhados são importantíssimos para a formação de todo profissional de saúde, uma vez que o preparam para lidar com diversas questões sociais, culturais e de gênero. Torna-se, pois, indispensável implementar a transversalidade desses conteúdos em outras disciplinas curriculares a fim de possibilitar a incorporação da integralidade do cuidado à formação profissional.

\section{REFERÊECIAS}

1. Aquino PS, Ximenes LB, Pinheiro AKB. Políticas públicas de saúde voltadas à atenção à prostituta: breve resgate histórico. Enferm Foco 2010;1(1):18-22.

2. Medeiros PF, Guareschi NMF. Políticas públicas de saúde da mulher: a integralidade em questão. Estud Fem 2009; 17(1):296

3. Souza MHN, Tyrrell MAR. Políticas de salud a la mujer en Brasil, 1974-2004. Rev Enferm UERJ 2011;19(1):70-76.

4. Coelho EAC, Fonseca RMGS, Garcia TR. Direitos sexuais e reprodutivos e saúde da mulher: conquistas e desafios. Salvador: Redor; 2006.

5. Alves AR, Lopes MF, Barletto M, Bevilacqua PD. Integralidade do cuidado em saúde sexual e reprodutiva: dificuldades da prática profissional. Oikos: Revista Brasileira de Economia Doméstica 2011;22(1):195-209.

6. Ministério da Saúde. Direitos Sexuais e Reprodutivos: uma prioridade do governo. Normas e Manuais Técnico. Brasília, DF: Ministério da Saúde; 2005.

7. Ferreira SL, Nascimento ER. Transversalidade de conteúdos nas diretrizes curriculares: o gênero no ensino da enfermagem. Rev Bras Enferm 2004;57(1):71-74.

8. Sá-Silva JR, Almeida CD, Guindani JF. Pesquisa documental: pistas teóricas e metodológicas. Rev Bras Hist Ciênc Sociais 2009; $1(1): 1-15$.

9. Minayo MCS. Fase de Análise ou Tratamento do Material In: O Desafio do Conhecimento- Pesquisa Qualitativa em Saúde. São Paulo: Hucitec-Abrasco; 2008.
10. Lessa, GM. Consciência de gênero e desenvolvimento no currículo de enfermagem. Salvador. Dissertação [Mestrado em Enfermagem] - Universidade Federal da Bahia; 1998.

11. Ferreira SL. O pensamento feminista e os estudos de gênero: esperi^ncias na Escola de Enfermagem da UFBA. Salvador: EDUFBA; 2012.

12. Christófaro MAC. Considerações da Associação Brasileira de Enfermagem sobre a proposta de reformulação do currículo mínimo para a formação do enfermeiro. In: Santos EF, Santos EB, Santana OS, Assis MF, Oliveira R. Legislação em Enfermagem: atos normativos do exercício e do ensino de enfermagem. São Paulo: Atheneu; 2002. p.248-250

13. Freire P. Pedagogia do oprimido. Rio de Janeiro: Paz e Terra; 2011

14. Freire P. Pedagogia da autonomia: saberes necessários à prática educativa. 43. ed. São Paulo: Paz e Terra; 2011.

15. Fonseca RMGS, Amaral MA. Reinterpretação da potencialidade das Oficinas de Trabalho Crítico-emancipatórias. Rev Bras Enferm 2012;65(5):780-787.

16. Oliveira EM. O Feminismo desconstruindo e re-construindo o conhecimento. Estud Fem 2008;16(1):229-245.

17. Bandeira L. A contribuição da crítica feminista à ciência. Rev Estud Fem 2008;16(1)1:207-228.

18. Tyrrel MAR, Santos AEV, Lucas EAJCF. Ensino de Enfermagem Obstétrica no Brasil: (des)acertos 1972-1996. Rev Bras Enferm 2005;58(6):677-681. 